\title{
Removal of Arsenic from Paint Industries Wastewater by Chemically Modified Low Cost Adsorbent Derived from Sugar Cane Bagasse
}

\author{
Melese Ayalew Yalew ${ }^{1} \quad$ Mariye Majo Shute ${ }^{1} \quad$ Tsehaye Yigzaw Tessema $^{1} \quad$ Alemayehu Kiflu $^{2}$ \\ Teka Girmay Hishe ${ }^{2}$ \\ 1.Department of Chemical Engineering, Technology Faculty, Debre Tabor University, Debre Tabor, Ethiopia \\ 2. Department of Chemical Engineering, Faculty of Chemical and Food Engineering, Bahir Dar University, \\ Bahir Dar Institute of Technology, Bahir Dar, Ethiopia \\ 3. Department of Hydraulic and Water Resources Engineering, Technology Faculty, Debre Tabor University, \\ Debre Tabor, Ethiopia
}

\begin{abstract}
Arsenic is one of the most toxic heavy metals to the environment which cause community health problem due to its high toxic nature. Several physiochemical techniques, such as adsorption, ion exchange, lime softening, reverse osmosis, coagulation and precipitation are used for arsenic removal but in this study, adsorption was used because of its simplicity to use and availability of a wide range of adsorbents. Adsorption is the process which is used for the removal of heavy metals from wastewater. Although there are Various adsorbents methods used to remove different type of heavy metal ions for study, iron impregnated activated carbon which was derived from sugarcane bagasse, was investigated as potential adsorbent for the removal of arsenic from aqueous solution. The synthetic wastewater was prepared in the laboratory to conduct the experiments. Batch experiments were conducted to obtain the optimum conditions for arsenic adsorption. Effect of experimental parameter such as $\mathrm{pH}$, adsorbent dose, and initial arsenic ion concentration was determined at constant contact time of 30min and atmospheric temperature. The optimum conditions obtained were, $\mathrm{pH} 7,1 \mathrm{mg}$ adsorbent dose and initial concentration of $20 \mathrm{mg} / \mathrm{L}$. Adsorption behavior could be described using the Langmuir and Freundlich isotherm models. Adsorption data was well fitted in Langmuir isotherm model. The maximum adsorption efficiency was found to be $96.5 \%$ at optimum experimental parameters of $\mathrm{pH} 7$, adsorbent dose of $1 \mathrm{~g}$ and initial arsenic concentration of $20 \mathrm{mg} / \mathrm{L}$.
\end{abstract}

Keywords: Chemical activation, Carbonization, Impregnation, Activated Carbon, Adsorption, Settling, Filtration, Arsenic Removal

DOI: $10.7176 / \mathrm{CER} / 12-10-03$

Publication date:October $31^{\text {st }} 2020$

\section{INTRODUCTION}

The presence of arsenic in the environment is ever-present in the world due to natural and anthropogenic sources. It exists in the earth's crust and is mobilized by natural weathering reactions, biological activity, geochemical reactions, and volcanic emissions[1].Concentrations as high as $5 \mathrm{mg} / \mathrm{L}$ of arsenic have been found in groundwater from arsenic-rich areas, and geothermal influences can increase arsenic levels, even up to $50 \mathrm{mg} / \mathrm{L}$. Mining, smelting, and the herbicide industries are the best examples of anthropogenic sources of arsenic pollution[2].

Elevated levels of arsenic are found in groundwater due to natural processes volcanic emissions, biological activities, burning of fossil fuels and weathering of arsenic bearing rocks and minerals and anthropogenic activities applications of arsenical pesticides, insecticides, wood preservatives, paints, drugs, dyes, semiconductors, incineration of arsenic containing substances, industrial wastewater discharge, mine tailing/landfill leaching[2] [2]. Naturally occurring arsenic in drinking water supplies affects over 137 million people in more than 70 countries; of which the most affected countries are Bangladesh, west Bengal (India), China, Chile, Argentina, Mexico, Hungry, Taiwan, and Vietnam. In order to minimize the health risk, the World Health Organization (WHO), United States Environmental Protection Agency (USEPA), and health Canada have reduced the maximum allowable contamination level (MCL) of total arsenic in drinking water from 10 to $50 \mu \mathrm{g} / \mathrm{L}$. This stringent regulation poses a major compliance challenge to the existing water supply systems[3].

Exposure to arsenic through drinking water sourced from groundwater poses a serious health hazard. Arsenic toxicity depends on its speciation, and the most significant forms for natural exposure of arsenic in drinking water are its inorganic forms. Long term drinking water exposure or chronic toxicity causes skin, lung, bladder, and kidney cancer as well as pigmentation changes, skin thickening hyperkeratosis neurological disorders, muscular weakness, loss of appetite, and nausea. This differs from acute poisoning, which typically causes vomiting, esophageal and abdominal pain, and bloody rice water diarrhea. High concentrations of arsenic in drinking water can also result in an increase in stillbirths and spontaneous abortions [4].

To overcome the foregoing problems, it is an urgent need to develop cost effective and technologically feasible systems to meet international environmental standard for arsenic. Adsorption is one of the most commonly 
used technologies to remove arsenic from water[5]. It is simple to perform and is usually inexpensive. Recent studies are focused on creating inexpensive and stable iron bearing adsorbents such as iron oxide coated sand, iron oxide impregnated activated carbon, GAC based iron containing adsorbent, GAC composites incorporated with iron/palladium (Fe/Pd) bimetallic Nano-particles [6].

GAC has large surface area, high pore volume, and rigid structure to be an ideal backbone for hosting a considerable amount of iron. Moreover, due to its ease of liquid/solid separation, GAC is widely used in water and advanced wastewater treatment facilities and is designated as the best available technology (BAT) by the U.S. EPA for the removal of organic compounds, odor and taste, and trace metals. But virgin activated carbon cannot be directly applied for arsenic treatment due to its lower arsenic adsorption. Research revealed that iron incorporated granular activated carbon can effectively remove arsenic from water without losing the capability of removing organic contaminants.

Millions of people in Western Bengal and Bangladesh have been drinking groundwater from wells that contain 100-2,000 $\mu \mathrm{g} / \mathrm{LAs}$, and many of those people have succumbed to diseases that are caused by the arsenic contaminated ground water. In the United State reported that over 35,000 people may drink contaminated with more than $50 \mu \mathrm{g} / \mathrm{L}$ of arsenic and over 2.5 million people could be supplied with water having arsenic levels over $25 \mu \mathrm{g} / \mathrm{L}$. Consumption of arsenic at the $50 \mu \mathrm{g} / \mathrm{L}$ level is estimated to cause mortality due to lung, kidney or bladder cancer in 1 out of every 1,000 or 10,000 people. The World Health Organization (WHO) announced that water containing more than $50 \mu \mathrm{g} / \mathrm{L}$ of arsenic is unsuitable due to acute and chronic toxicity. Owing to epidemiological evidence linking arsenic and cancer, the safe limit of arsenic in drinking water was reduced from $50 \mu \mathrm{g} / \mathrm{L}$ to 10 $\mu \mathrm{g} / \mathrm{Lin} 1993$ by WHO[7].

The focus of this research has been to discern a less expensive means of removing arsenic from groundwater, particularly for small municipalities. Compared to conventional Fe oxide media that are synthesized by the solgel process, the method of preparing iron tailored GAC herein is more environmentally acceptable, cost-effective, and simple; due to fewer preparation steps and less iron precursor. Moreover, there need be no waste left after media synthesis.

\section{MATERIALS AND METHODS}

\subsection{Preparation of Absorbent from sugarcane}

Sugarcane bagasse was collected around kebele 3 Bahir Dar Town, Bahir Dar Ethiopia. Raw bagasse was thoroughly washed with tap water to remove contaminants and then boiled in distilled water for one hour to remove the sugar residue in the bagasse. The washed bagasse was exposed to sunlight for three days, and then dried in an oven at $85{ }^{\circ} \mathrm{C}$. The dried bagasse was well crushed with a disk mill and sieved with $0.2 \mathrm{~mm}$ sieve to obtain a uniform grain size. Finally, the sieved material was labeled as raw bagasse pith (RBP) and stored in vacuum desiccator for further use. Chemical activation the raw bagasse pith (RBP) was soaked with 30\% phosphoric acid (H3PO4), stirred for 30minutes and kept for 24 hours. In treatment process, raw bagasse was fully immersed in acid with the ratio of $1 \mathrm{~g}$ bagasse to $30 \mathrm{ml}$ acid. The purpose of treating sugarcane bagasse with phosphoric acid was to create a suitable environment for ring opening of the bagasse, which increase the number of adsorption sites. The treated bagasse was washed with distilled water to remove all traces of the acid until it reached $\mathrm{pH}$ of 6.6 , then it is dried with an oven ((PH-030A, Germany) at $100^{\circ} \mathrm{C}$. Thermal activation the dried activated bagasse was carbonized in a furnace (LT3/12/B180) at 500oC for $1 \mathrm{hr}$. Produced activated carbon was reduced in size with mortar. The carbonized material was labeled as sugarcane activated carbon (SAC) and stored in vacuum desiccator for further use. For the preparation of iron impregnated sugarcane carbon (Fe-SAC), a solution of ferric oxide was kept at $70^{\circ} \mathrm{C}$ and $\mathrm{pH} 9.5$ for $10 \mathrm{~min}$ with the gentle stirring of $60 \mathrm{rpm}$ and then the solution was impregnated into the sugarcane activated carbon (SAC) and kept for 30minutes. Iron impregnated sugarcane activated carbon (Fe$\mathrm{SAC}$ ) was then washed by $0.01(\mathrm{M}) \mathrm{HCl}$ solution to remove all salts precipitated in its pores. Then, the Fe-SAC was repeatedly washed with distilled water to remove all traces of the acid. Subsequently, the washed Fe-SAC was oven-dried at $100{ }^{\circ} \mathrm{C}$ for $24 \mathrm{hrs}$ to volatilize the organic impurities. Finally, the dried Fe-SAC was stored in a vacuum desiccator for subsequent use as adsorbent.

\subsection{Characterization of adsorbent}

The absorbent characterization was done by taking Moisture content, Total ash content, Pore volume determination, Surface area (Methylene blue method), $\mathrm{pH}$ point of zero charge and Fourier Transform infrared (FTIR) and calculated using the following equations.

The moisture content was calculated by using the following formula [8].

$$
\text { Moisture content }(\%)=\frac{I-F}{I} * 100 \quad \text { Equation } 1
$$

Where I = Initial weight of the adsorbent taken for oven-drying (g);

$\mathrm{F}=$ Final weight of the adsorbent after drying $(\mathrm{g})$. 


\section{Total ash content}

The ash was then weighed and the ash content of the adsorbent was calculated as follows [24].

Total ash content $(\%)=\frac{D-B}{C-B} * 100$

Equation 2

Where $\mathrm{B}$ is the weight of the crucible $(\mathrm{g}), \mathrm{C}$ is the weight of the crucible plus original adsorbent $(\mathrm{g}) \mathrm{D}$ is the weight of the crucible plus ash containing adsorbent $(\mathrm{g})$.

\section{Pore volume determination}

The pore volume of the adsorbent was determined using the formulas:

$$
=\frac{W f-W I}{W I} * \text { density of water } \quad \text { Equation } 3
$$

For the determination of WI and Wf, $3 \mathrm{~g}$ of adsorbent was weighed into a beaker and the weight of sample plus beaker was noted as WI. Distilled water of $50 \mathrm{~mL}$ was added into the beaker containing the dry sample, and the mixture was boiled for $15 \mathrm{~min}$. After displacement of air from the pores, the sample was drained and then dried superficially and weighed. The weight was recorded as WF and procedure was repeated twice[8].

Surface area (Methylene blue method)

Methylene blue (MB), a cationic dye, has been used to determine the surface area of adsorbents for several years. Precision can be improved by means of accurate analytical techniques to determine the amount of absorbed MB. The value of surface area can be determined by calculation using the formula given below.

The specific surface area was computed from the amount of adsorbed MB at the optimum point[9].

$$
\text { Surface area; } S S A=\frac{m * A_{V} * A_{M B}}{M} \quad \text { Equation } 4
$$

Where $\mathrm{m}$ is the mass of the absorbed Methylene blue at the point of complete cat ion replacement, $\mathrm{mS}$ is the mass of the adsorbent specimen, Av is Avogadro 's number $(6.02 \times 1023 / \mathrm{mol}-1)$, AMB is the area covered by one MB molecule (typically assumed to be 197.2A2) and 319.87 is the corresponding molecular weight of MB.

pH point of zero charge

The point of zero charge (pHpzc) of the adsorbent was determined by the solid addition method. A 50mL of $0.1(\mathrm{M})$ KNO3 solution was added into three $100 \mathrm{~mL}$ conical flasks. The initial $\mathrm{pH}(\mathrm{pH} 0)$ values of the solutions were adjusted from 1.0 to 10.0 by adding $0.1 \mathrm{~N} \mathrm{HNO} 3$ and $0.1 \mathrm{~N} \mathrm{KOH}$ using a pH meter. Then $1.5 \mathrm{~g}$ of adsorbent was added to each conical flask which was securely capped immediately. The flasks were then placed into constant temperature water bath shaker and shaken for $24 \mathrm{hr}$. The $\mathrm{pH}$ values of the supernatant liquid were noted after $24 \mathrm{hr}$. The value of pHpzc could be found from the curve that cuts the line of $\mathrm{pHi}$ of the plot of pHf versus pHi, (pHpzc)[9]. Fourier Transform infrared (FTIR)

spectroscopy Infrared (IR) spectroscopy measures the absorption of IR radiation by materials as the atoms vibrate about their bonds. It is a routine tool for detecting functional groups, identifying compounds, and analyzing mixtures.

IR sensitive vibrations are associated with changes in dipole moments occurring during the vibration. The infrared region of the electromagnetic spectrum may be divided into three main sections: Near-infrared (overtone region); $0.8-2.5 \mu \mathrm{m}(12500-4000 \mathrm{~cm}-1)$, Middle-infrared (vibration-rotation region); $2.5-50 \mu \mathrm{m}(4000-200 \mathrm{~cm}-$ 1) and Far-infrared (rotation region); $50-1000 \mu \mathrm{m}(200-10 \mathrm{~cm}-1)$. The main region of interest for analytical purpose is from 2.5 to $25 \mu \mathrm{m}$ i.e., wave number 4000 to $400 \mathrm{~cm}-1$.

When the sample is illuminated by IR radiation of the appropriate frequencies; atoms, ions, and functional groups in molecules will vibrate about their bonds and energy will be absorbed. Each bending and stretching vibration mode of a molecule or functional group will absorb at a particular frequency. When exposed to appropriate IR frequencies, energy will be absorbed from the incident radiation as vibration intensities increase. Many IR frequencies have no effect at all and will not be absorbed[9].

In this study, IR characterization of the adsorbents were carried out with Fourier Transform Infrared spectrophotometer (Perkin-Elmer, FTIR, Model-RX1 Spectrometer, USA) in the range of 400-4000 cm-1. Adsorbent samples were prepared in the forms of potassium bromide (KBr) disk. The adsorbents in the form of powder or finely divided states were ground with $200 \mathrm{mg}$ of $\mathrm{KBr}$ (spectroscopic grade) in a mortar and pressed into $10 \mathrm{~mm}$ diameter disks less than 10 tons of pressure and high vacuum for FTIR analysis.

\subsection{Preparation of stock solution}

The stock solution of As (V) having concentration $10 \mathrm{mg} / \mathrm{L}, 20 \mathrm{mg} / \mathrm{L}$ and $30 \mathrm{mg} / \mathrm{l}$ was prepared as model waste water by dissolving $\mathrm{Na} 2 \mathrm{HAsO} 4 \cdot 7 \mathrm{H} 2 \mathrm{O}$ in three $1000 \mathrm{ml}$ flasks with distilled water. The $\mathrm{pH}$ of the solutions was adjusted to desired value by using $0.1 \mathrm{NaOH}$ and $0.1 \mathrm{HCl}$ solutions using digital $\mathrm{pH}$ meter ( $\mathrm{pH}-016)$ before adsorption.

\subsubsection{Adsorption experimental setup}

In the batch adsorption experiments, the effect of different parameters; $\mathrm{pH}$, initial arsenic concentration and dose of adsorbent on adsorption of arsenic was studied in three levels. The $100 \mathrm{~mL}$ of arsenic solution with concentration of $(10 \mathrm{mg} / \mathrm{L}, 20 \mathrm{mg} / \mathrm{L}$ and $30 \mathrm{mg} / \mathrm{L})$ was taken in flasks of volume $250 \mathrm{~mL}$ separately. Known quantity of dried 
adsorbent $(0.5 \mathrm{~g}-1.5 \mathrm{~g})$ was added in each flask. The $\mathrm{pH}$ was adjusted from $\mathrm{pH} 4-\mathrm{pH} 10$ with $0.1 \mathrm{M}$ of $\mathrm{NaOH}$ and $\mathrm{HCl}$ and the arsenic bearing suspensions was kept under magnetic stirring for constant time of 30 minutes at ambient temperature until the equilibrium was reached. After stirring, the suspension was allowed to settle down for $2 \mathrm{hrs}$ and filtered using Whatman-42 grade filter paper using vacuum filter (CAPEXL2C). The filtrate was collected and subjected for arsenic estimation using atomic absorption spectrophotometer (novAA400p). The arsenic concentrations of each run before and after adsorption were recorded, and then the adsorption capacity and removal efficiency of arsenic was determined. The effect of initial concentration, adsorbent dose, and $\mathrm{pH}$ as well as interaction effects of the parameters were analyzed by factorial design using Design Expert 6.0.8 software.

\subsection{Experimental frame work of arsenic removal}

\subsection{Experimental frame work}

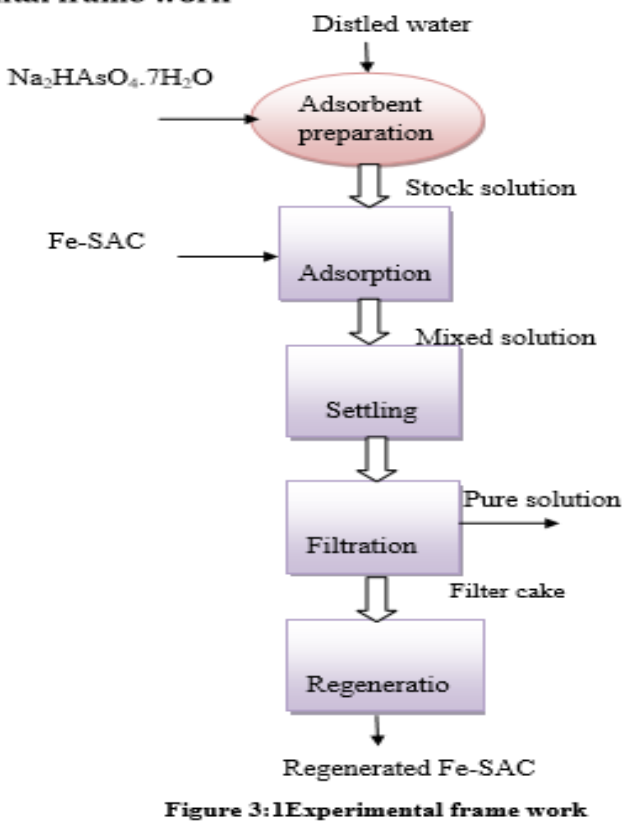

Figure 1 experimental frame work

\section{RESULTS AND DISCUSSION}

From the experiment Pore volume Pore volume indicates that a fraction of pores in the granular activated carbon of the adsorbent media (Fe-SAC) was found $2.01 \mathrm{~cm}^{3} / \mathrm{g}$. The optimum amount of Methylene blue adsorbed was $46.5 \mathrm{mg} / \mathrm{g}$ and the surface area of iron modified sugar cane activated carbon was $803.12 \mathrm{~m} 2 / \mathrm{g}$. The point of zero charge which means the point that $\mathrm{pH}$ does not change i.e. the point at which the initial and final $\mathrm{pH}$ is the same is defined as the $\mathrm{pH}$ of point of zero charge (pHpzc). In Figure 4.3, the red line represents the experimental values. The intersection point of the two curves is the point of zero charge (pHpzc) the point of zero charge of Fe-SAC was 6.32 for $1 \mathrm{~g}$ adsorbent. The point of zero charge of an adsorbent is a significant parameter which contributes to the types and intensity of adsorption. At lower $\mathrm{pH}$, the activated carbon begins to dissolve, thus losing adsorptive capacity. At higher $\mathrm{pH}$, activated carbon loses its positive charge, limiting electrostatic attraction between the positive surface and negative arsenate. Also, at higher $\mathrm{pH}$, there is an increase in $\mathrm{OH}$-in solution, which competes with arsenate for adsorption sites[10] [13]. Fourier Transform infrared (FTIR) spectroscopy which was analyzed to determine the surface chemistry of the adsorbent Fe-SAC) is also very important step. The FTIR spectra of FeSAC were characterized before and after adsorption and the result is presented in the figure below. The percentage transmittance of the adsorbent was increased after adsorption. This is indicating the surface of the adsorbent was occupied by arsenic ions. The peaks observed from $375-3150 \mathrm{~cm}^{-1}$ may be due to the stretching of C-H and $\mathrm{O}-\mathrm{H}$ groups of alkyl and carboxylic acid whereas the peaks observed from $1380-1740 \mathrm{~cm}^{-1}$ may be due to the stretching of $\mathrm{C}=\mathrm{C}$ and $\mathrm{C}=\mathrm{O}$ groups in the presence of aromatics and ketone. The peaks observed from 1055-1392 cm-1 can be due to stretching of aromatic primary amine $(\mathrm{C}-\mathrm{N})$, plane deformation of aromatic compounds $(\mathrm{C}-\mathrm{H})$ and by $\mathrm{N}-\mathrm{H}$ stretching of primary aromatic amine.

The physical-chemical properties (moisture content, total ash content, pore volume, specific surface area and $\mathrm{pH}$ point of zero charge ( $\mathrm{pHpzc})$ are summarized in the table below. 
Table 1 physico chemical charactersitics of Fe-SAC adsorbent

\begin{tabular}{ll}
\hline Parameters & Value \\
\hline Moisture content (\%) & $8.03 \pm 0.2$ \\
Total ash content (\%) & $6.66 \pm 0.1$ \\
Bulk density $\left(\mathrm{g} / \mathrm{cm}^{3}\right)$ & $0.428 \pm 0.03$ \\
Pore volume $\left(\mathrm{cm}^{3} / \mathrm{g}\right)$ & $2.01 \pm 0.0 .05$ \\
Specific surface area $\left(\mathrm{m}^{2} / \mathrm{g}\right)$ & $803.12 \pm 3.5$ \\
$\mathrm{pH}$ point of zero charge & $6.32 \pm 0.12$
\end{tabular}

\subsection{Batch adsorption}

The removal efficiency of arsenate As (V) varied from run to run even if the adsorbent is the same. This is due to the effect of process parameters on adsorption. The adsorption process depends on the amount of initial concentration, adsorbent dose and $\mathrm{pH}$. Due to this, the interaction effects of those parameters were studied in the experiment with two replications for each run. The percentage removal efficiency was determined by taking the average weight of arsenic adsorbed divided by the corresponding initial arsenic concentration. The average percentage removal efficiency of arsenic was calculated as follows for each run.

Average removal efficiency $(\%)=\frac{\mathrm{C}_{\mathrm{i}}-\mathrm{C}_{\mathrm{e}}}{\mathrm{C}_{\mathrm{i}}} * 100 \quad$ Equation 5

Where $\mathrm{Ci}=$ initial arsenic concentration and $\mathrm{Ce}$ is equilibrium concentration.

The data was analyzed by Design Expert (version 6.0.8) software. The effect of process parameters and their interactions on the response variable are evaluated by general factorial methodology (GFM), the main factors and their significant effects have been determined.

\subsubsection{Effect of initial arsenic concentration}

The initial concentration of arsenic ions in the solution is an important parameter as the concentration changes over a broad range in different aqueous solutions. In this research, the adsorption behavior of arsenic was studied in the concentration range of $10-30 \mathrm{mg} / \mathrm{L}$ at $\mathrm{pH} 7$ and adsorbent dose of $1 \mathrm{~g}$ with varying initial arsenate concentration from 10 to $30 \mathrm{mg} / \mathrm{L}$ to find out the influence of initial arsenate concentration on adsorption. The experiment has been done at constant contact time of 30min with atmospheric temperature. The removal efficiency depends on the number of arsenic ions present in the adsorbate. The result was illustrated in figure 2.

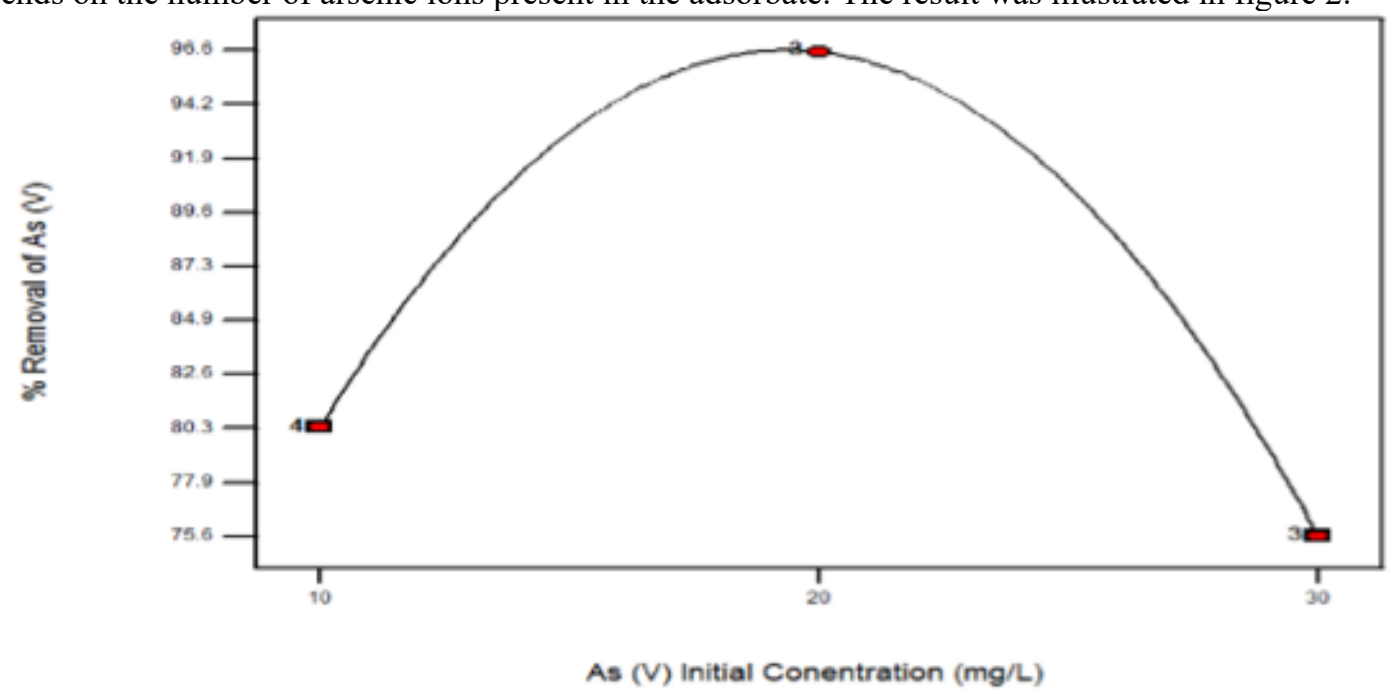

Figure 2 effects of initial $\mathrm{AS}(\mathrm{V})$ concentration on removal efficiency

Figure 2 Illustrated that, the change in adsorption trend of arsenic on Fe-SAC adsorbent with variation in initial concentration of adsorbent in the solution. The percentage removal of arsenic on Fe-SAC adsorbent was initially increased from $80.3 \%$ to $95.8 \%$ with increasing initial concentration reaching the optimum level of $95.8 \%$ at $20 \mathrm{mg} / \mathrm{L}$. Thereafter, the percentage removal showed sharp decrease to $75.6 \%$ as the initial concentration increased to $30 \mathrm{mg} / \mathrm{L}$.

This significant increase in percentage removal of arsenic can be attributed due to the presence of more 
unoccupied adsorption sites on the surface of the adsorbent when the concentration was low so that the uptake of As (V) increased. This is because at lower initial arsenic concentrations, sufficient adsorption sites are available for adsorption of As (V) ions. On the other hand, even if, the interaction of arsenic species with the available active sites on the adsorbent surface could be higher at higher initial concentration due to increased diffusion and mass transfer, the percentage removal of As (V) on Fe-SAC clearly showed the decreasing trend with increasing of concentration. It can be attributed that at higher concentrations of $\mathrm{As}(\mathrm{V})$, relatively less available active sites of Fe-SAC induced reduction in adsorption of As (V) on the adsorbent surface due to the saturation of free adsorption sites and lack of available active sites on the adsorbent surface.

For a given adsorbent dose the total number of adsorbent sites available is fixed, thus adsorbing different amount of adsorbate with fixed number of active site consequent the decrease in the removal capacity of adsorbate. This is because, for a fixed dose of the adsorbent, the total available binding sites are limited, thereby binding the same amount of metal ions. This resulted in a decrease in percentage removal of the metal ions as the initial metal ion concentration increases. Therefore, it was evident from the results that arsenic adsorption was dependent on the initial arsenic concentration. Similar trends were reported by many authors using different types of adsorbents. Hence high adsorption efficiency of As (V) was achieved at optimum concentration $(20 \mathrm{mg} / \mathrm{L})$ of arsenic by using Fe-SAC $[10,11]$.

However, the adsorption capacity has been decreased thorough out the increment of the initial concentration. The adsorption behavior in fig.4.6 clearly indicated the increasing trend of adsorption capacity on Fe-SAC from 8.3 to $27.6 \mathrm{mg} / \mathrm{g}$ as the initial As (V) concentration increased from 10 to $30 \mathrm{mg} / \mathrm{L}$. The increase in adsorption capacity with increasing of initial concentration may be due to the utilization of all active sites available for the adsorption at higher concentration and higher availability of As (V) ions in the solution for the adsorption.

\section{Effect of adsorbent dose}

The amount of adsorbent added in the aqueous solution is an important parameter in adsorption process. In this research, the adsorption efficiency of As (V) was studied by varying the amount of adsorbents from 0.5 to $1.5 \mathrm{~g}$ keeping other process parameters constant ( $\mathrm{pH}-7$ \& initial concentration of $20 \mathrm{mg} / \mathrm{L}$ ). The effect of adsorbent dose on the adsorption efficiency of As (V) was illustrated in Figure3.

From the figure, it can be seen initially gradual increase in As (V) adsorption percentage was observed with increasing adsorbent dose. The maximum arsenic adsorption was achieved using $1 \mathrm{~g}$ of adsorbent dose. The adsorption efficiency rapidly increased from $80.3 \%$ to 96.3 with increasing of adsorbent dose from 0.5 to $1 \mathrm{~g}$ and then, it was slightly increased to 96.5 with further increasing of adsorbent dose to $1.5 \mathrm{~g}$.

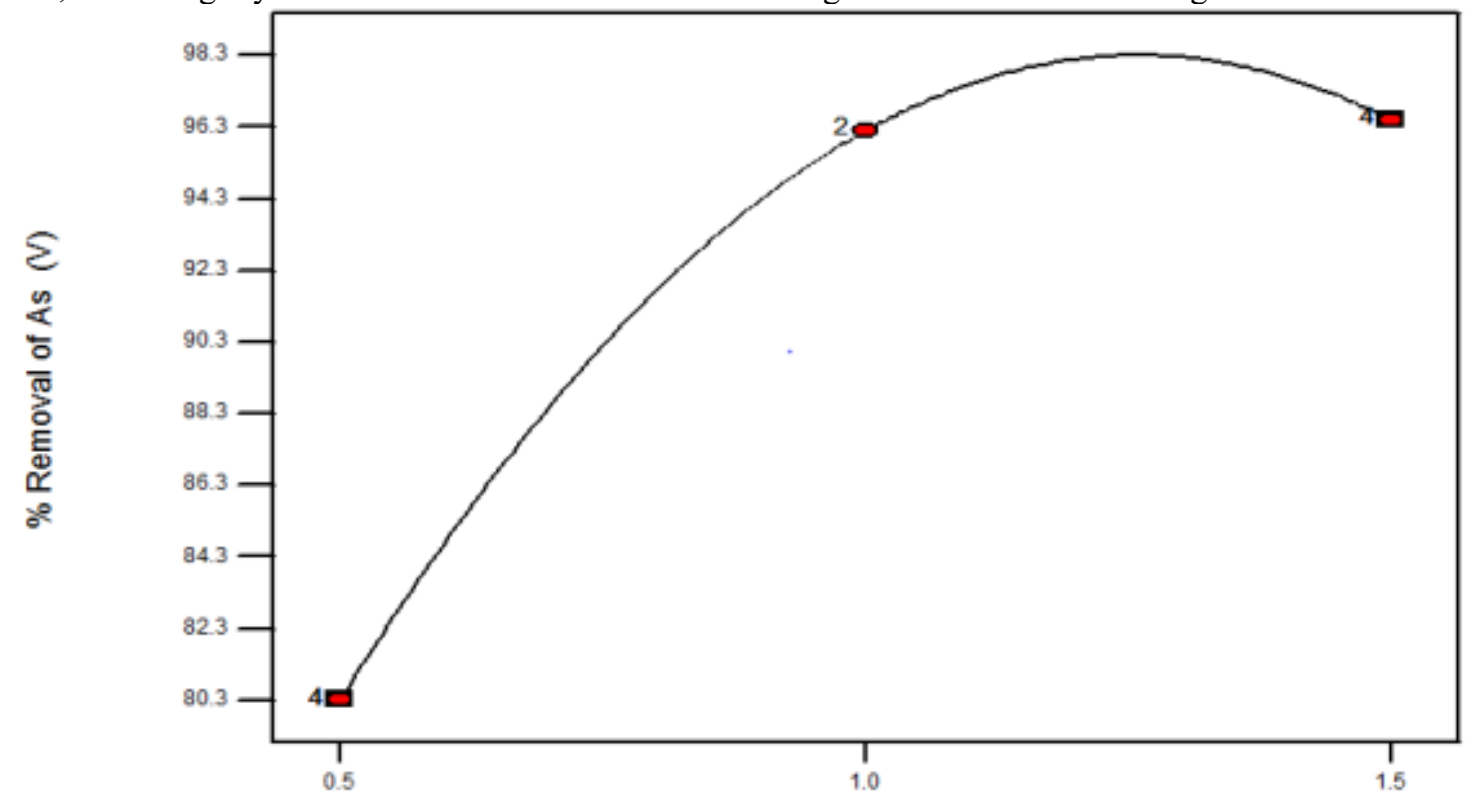

\section{Figure 3 Effect of adsorbent dose on adsorption efficiency}

Adsorbent dose $(g)$

The low adsorption percentage at lower adsorbent dose $(0.5 \mathrm{~g})$ can be attributed to the fact that the adsorbent has a lower number of active sites that would have achieved saturation above a certain adsorbate concentration. The rapid increase in As (V) removal with increasing in adsorbent dose to $1 \mathrm{~g}$ was due to the increment of vacant adsorption sites. As the adsorbent dose increased, the number of adsorbent active sites increased and hence more As (V) ions attached to the adsorption sites. At the dose of $1 \mathrm{mg}$, the available active sites in adsorbent were sufficient, thus, the adsorption continues the increasing trend reaching the maximum removal at $1 \mathrm{~g}$ [12]. 
A negligible change was observed in percentage adsorption of As (V) with increasing in adsorbent dose beyond $1 \mathrm{~g}$. This is because of that, almost all the vacant active sites presented in the adsorbent were filled up with the ion at prolonged time. At this particular adsorbent dose, the percentage adsorption has reached equilibrium by maintaining constant initial As (V) concentration. Hence the number of ions bound to the adsorbent and the number of free ions in the solution remains constant. Thus, adsorbent dose of $1 \mathrm{~g}$ was considered as optimum[10]. On the other hand, the adsorption capacity of the adsorbent was decreased with increasing the adsorbent dose as it can be seen in fig.4.8. From the figure, it was illustrated that, the adsorption capacity of the adsorbent was decreased from 19.2 to $18.3 \mathrm{mg}$ of As (V) per gram of adsorbent as the adsorbent dose was increased from $0.5 \mathrm{~g}$ to $1 \mathrm{~g}$, then it was slightly decreased to $17.8 \mathrm{mg} / \mathrm{g}$ with further increasing of adsorbent dose to $1.5 \mathrm{~g}$. The decrease in adsorption capacity is due to the fact that, as the amount of the adsorbent increased, the amount of active sites remaining unsaturated increased during the adsorption process. This can be explained as the amount of the adsorbent increased for constant adsorbate concentration, the ration of the mass of the adsorbed ions to the mass of adsorbent is decreased.

\subsubsection{Effect of pH}

The influence of various process parameters on adsorption of pollutants from aqueous phase has been well established. Solution $\mathrm{pH}$ is one of the important process parameters that significantly affect the adsorption of arsenic on adsorbent. In this study, the adsorption of arsenic was studied at different $\mathrm{pH}$ values ranging from 4-10 to determine the effect of $\mathrm{pH}$ on adsorption behavior of arsenic onto the Fe-SAC adsorbent. The experiments were performed at definite experimental conditions (initial arsenic concentration of $10 \mathrm{mg} / \mathrm{L}$ and adsorbent dose of $1 \mathrm{~g}$ ), by varying the $\mathrm{pH}$ from 4 to 10 . This study was done at constant contact time of 30 and atmospheric temperature. The results indicated that, the percentage removal efficiency of As (V) was increased from 83.5 to $95.1 \%$ as the $\mathrm{pH}$ level was increased from acidic condition to neutral (4 to 7) and then it is decreased to $80 \%$ with increasing $\mathrm{pH}$ to 10 basic conditions.

Similarly, the adsorption capacity of Fe-SAC for As (V) is increased from 17.8 to $19.2 \mathrm{mg} / \mathrm{g}$ with increasing the $\mathrm{pH}$ level from 4 to 7 as it can be seen in fig. 4.10 and then it is decreased to $17.4 \mathrm{mg} / \mathrm{g}$ when the $\mathrm{pH}$ is increased to 10 .

The dependence of As (V) adsorption on $\mathrm{pH}$ can largely be related to type and ionic state of the functional groups present on the surface of the adsorbent as well as the metal chemistry of the adsorbate. Activated carbons were considered as amphoteric solids due to the existence of variety of surface functional groups. Arsenic can be found in both cation and anion forms since it has metalloid property so that its removal efficiency depends on the surface groups of the adsorbent. Under acidic conditions, the surface of the adsorbent was positively charged and it seemed reasonable that the neutral or negatively charged arsenic species were absorbed by electrostatic attraction force. When the solution $\mathrm{pH}$ has been decreased, the repulsion between positively charged adsorbent surface and positively charged arsenate ions was increased. Therefore the adsorption efficiency was low due to the competition between the proton $(\mathrm{H}+)$ and arsenate $(\mathrm{As} 5+)$ ions for same functional groups which resulted in a higher electrostatic repulsion between surface and metal ions. When the $\mathrm{pH}$ of the solution increased to $\mathrm{pH} 7.0$, the solution became neutral and more arsenic cat ions (As5+) were easily absorbed by the active surfaces of the adsorbent because of the decrease in competition between proton and metal cations so that the percentage removal has been increased. However, as the $\mathrm{pH}$ has been increased to basic condition, the degree of protonation of the adsorbent decreased gradually and the amount of $\mathrm{OH}$ ions increased which, resulted in low adsorption of As (V) due to the competition between $\mathrm{OH}-$ and As5- ions so that the adsorption efficiency has been decrease [10]. Therefore, $\mathrm{pH}$ dependent behavior of arsenate adsorption onto the adsorbent was the consequence of interaction of the aqueous arsenic with the charged surface of the adsorbent. The speciation of arsenic is a function of $\mathrm{pH}$ and the charge on the adsorbent surface is a function of $\mathrm{pH}$ at its point zero charge ( $\mathrm{pHzpc}$ ), at which the net surface charge is zero. The species of arsenate exist as $\mathrm{H} 2 \mathrm{AsO} 4-, \mathrm{HAsO} 42-$ and AsO43-when the $\mathrm{pH}$ shifts from acidic to alkaline condition corresponding to their dissociation constants: $\mathrm{pKa} 1=2.3, \mathrm{pKa} 2=6.8, \mathrm{pKa} 3=11.6[12]$. It is well known that the adsorbent surface is positively charged at $\mathrm{pH}$ below $\mathrm{pHPZC}$ and negatively charged at $\mathrm{pH}$ above pHPZC, resulting in increased electrostatic attraction or repulsion with anionic species. The surface of the adsorbent becomes less positively charged when $\mathrm{pH}$ increases and thus shows less attraction towards anionic arsenate species. Therefore, the adsorption of arsenate ions was found mainly to be influenced by solution $\mathrm{pH}[12]$ [11].

\subsection{Analysis of interaction effects}

The contour plots of interaction of factors were shown in Figure 4.10. Compared to the effects of main factors, the effect of interaction of factors on the response is less. The interaction effects $\mathrm{pH} *$ concentration $(\mathrm{AB})$ and concentration * adsorbent dose (AC) and $\mathrm{pH}^{*}$ adsorbent dose $(\mathrm{BC})$ are considered to be significant for As (V) adsorption on Fe-SAC.

\subsubsection{Interaction effects of concentration and adsorbent dose}

The interaction graph between concentration and adsorbent dose below is shown that the removal efficiency was 
increased with increasing the amount of adsorbent to the maximum efficiency of 96.5. However, it was decreased as the amount of adsorbent is decreased and that of concentration is increased and also the efficiency is decreased when both concentration and adsorbent dose were decreased.

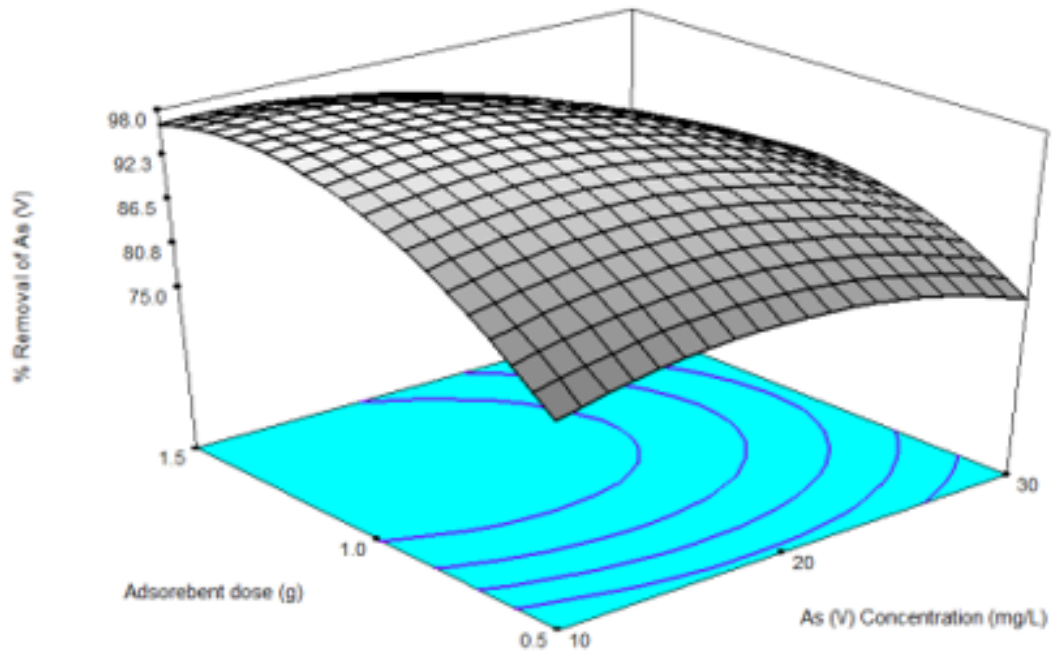

Figure 4 Integration effect of concentration and adsorbent dose

\subsubsection{Interaction effects of concentration and $\mathbf{p H}$}

From figure 5, it can be seen that the removal efficiency was initially increased as both concentration and pH were increased and then it was decreased as concentration and $\mathrm{pH}$ ware further increased beyond the optimum values. The maximum percentage removal efficiency $(95.6 \%)$ was achieved at the center of the 3D surface at the optimum conditions of concentration $20 \mathrm{mg} / \mathrm{L}$ and $\mathrm{pH}-7$.

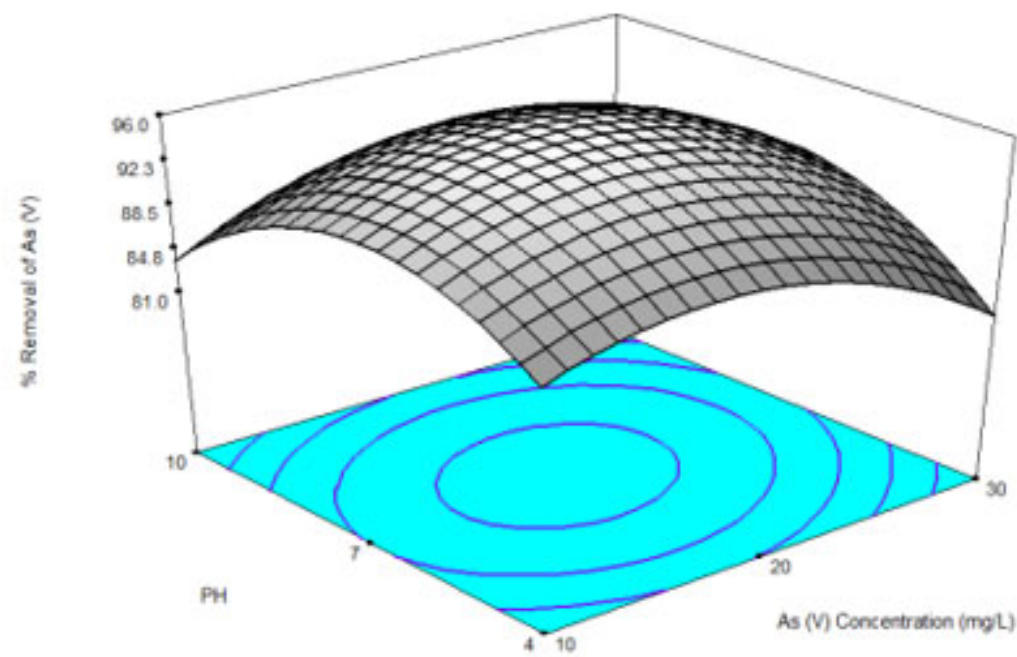

Figure 5 interaction effects of concentration and pH

\subsubsection{Interaction effects of adsorbent dose and $\mathrm{pH}$}

The interaction effect of adsorbent dose and $\mathrm{pH}$ on the adsorption efficiency of As (V) by FeSAC was illustrated in figure6. From the figure, it can be seen that, the percentage removal efficiency of As (V) was increased from 81.4 to $95.3 \%$ as the $\mathrm{pH}$ level was increased from acidic condition to neutral (4 to 7 ) and then decreased to $76.2 \%$ with increasing $\mathrm{pH}$ to 10 (basic conditions). Similarly, As (V) adsorption percentage was increased from 76.2 to $96.3 \%$ with increasing adsorbent dose from 0.5 to $1 \mathrm{~g}$ and decreased $83.2 \%$ as the amount of adsorbent was increased to $1.5 \mathrm{~g}$. The maximum adsorption efficiency was achieved at the center of the 3D surface at the optimum conditions of adsorbent dose of $1 \mathrm{~g}$ and $\mathrm{pH}-7$. 


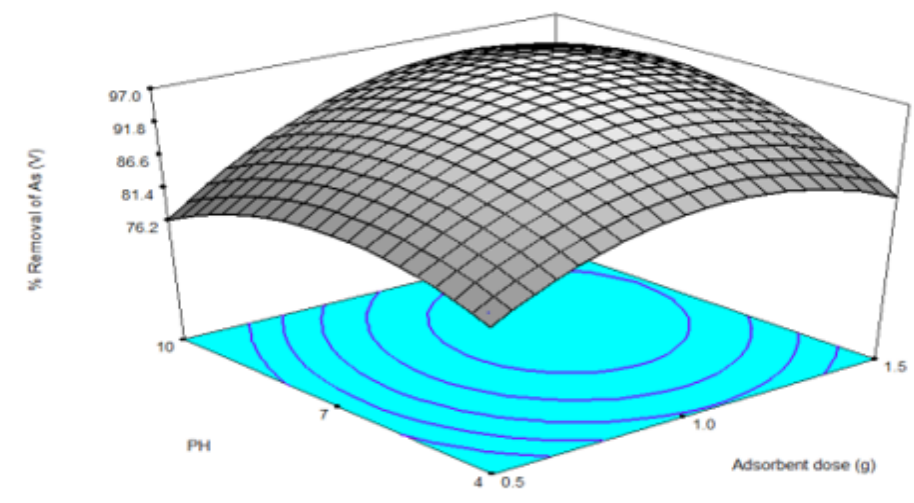

Figure 6 Interaction effects of pH and Adsorbent Dose

\section{CONCLUSION}

The adsorptive removal of arsenic from aqueous solutions, by utilizing iron impregnated activated carbon derived from sugarcane bagasse (Fe-SAC) was investigated at different initial ion concentration, adsorbent dosage and $\mathrm{pH}$ levels. To assess the potentiality of the adsorbent, the influence of process parameters such as $\mathrm{pH}$, adsorbent dosage, and initial metal ion concentration on the removal efficiency were studied with batch adsorption experiments. The study showed that all this process parameters affect the removal efficiency. Design expert 6.0.8 model was used to show the validity of Fe-SAC as a preferable adsorbent for arsenic removal. The study showed that in batch operations, removal efficiency increased from $80.3 \%$ to $95.8 \%$ with increasing in initial arsenic concentration from $10 \mathrm{mg} / \mathrm{L}$ to $20 \mathrm{mg} / \mathrm{L}$ and removal efficiency decreased to $75.6 \%$ as the concentration was increased to $30 \mathrm{mg} / \mathrm{L}$ due to over dosing of Arsenic concentration. The adsorption efficiency of the adsorbent was increased from $80.6 \%$ to $96.3 \%$ when the adsorbent dose was increased from $0.5 \mathrm{~g}$ to $1 \mathrm{~g}$ and then further increasing of the adsorbent to $1.5 \mathrm{~g}$ has been slightly increased percentage arsenic removal to $96.5 \%$.

The study also showed that adsorption efficiency of Fe-SAC for As (V) at $\mathrm{pH} 4$ was $83.5 \%$ and it was increased to $95.1 \%$ as $\mathrm{pH}$ increased to $\mathrm{pH} 7$. The removal efficiency of Fe-SAC was then gradually reduced to $80 \%$ when the $\mathrm{pH}$ has been further increased to $\mathrm{pH} 10$.

The optimum initial concentration of Arsenic, adsorbent dose and $\mathrm{pH}$ of solution for arsenic removal were found to be $20 \mathrm{mg} / \mathrm{L}, 1 \mathrm{~g}$ and 7 respectively. Under these optimum conditions, the arsenic uptake capacity and the maximum removal efficiency of Fe-SAC were found to be $19.3 \mathrm{mg} / \mathrm{g}$ and $96.5 \%$ respectively.

The adsorption data were tested to Langmuir and Freundlich isotherm models and this study revealed that Langmuir isotherm model gave the best fit to this batch experimental data. The data for Fe-SAC fit well with Langmuir isotherm model. The kinetics of adsorption was studied by pseudo first order and pseudo second order models and the result showed that, the pseudo second order model was fit more than pseudo first order. From this study out comes it can be concluded that Fe-SAC powder can be used for arsenic removal from aqueous solution.

\section{Acknowledgment}

First of all, we would like to thank our God for giving us initiation to start and the strength and life to finish this thesis research. We would like to express our sincere gratitude to our advisor Dr. Alemayehu Kiflu for his guidance, supervision, precious suggestions and insightful advice throughout this challenging journey that enabled us to prepare this study. We need to thank Dr. Nigus Gabbiye, scientific director of Bahir Dar Institute of Technology; Dr. Zenamarkos Bantie, head of chemical and faculty, for allowing us to utilize the lab facility in the department. Our sincere thanks are also to members of Doctoral Scrutiny Committee and faculty members, staffs of Chemical Engineering Department for their suggestions and constructive criticism during our research work. Last but not least, we would like to thank all friends for their consistent discussions, suggestions sharing ideas and encouragement during this study.

\section{REFERENCE}

1. Jain, C. and I. Ali, Arsenic: occurrence, toxicity and speciation techniques. Water research, 2000. 34(17): p. 4304-4312.

2. Jang, M., W. Chen, and F.S. Cannon, Preloading hydrous ferric oxide into granular activated carbon for arsenic removal. Environmental science \& technology, 2008. 42(9): p. 3369-3374.

3. Kalderis, D., et al., Adsorption of polluting substances on activated carbons prepared from rice husk and sugarcane bagasse. Chemical engineering journal, 2008. 144(1): p. 42-50.

4. Kang, S.-Y., D.-W. Kim, and K.-W. Kim, Enhancement of As (V) adsorption onto activated sludge by methylation treatment. Environmental geochemistry and health, 2007. 29(4): p. 313-318. 
5. Mohammed, A., et al., A comparative analysis and characterization of animal bones as adsorbent. Adv. Appl. Sci. Res, 2012. 3(5): p. 3089-3096.

6. Krishnan, K.A., K. Sreejalekshmi, and R. Baiju, Nickel (II) adsorption onto biomass based activated carbon obtained from sugarcane bagasse pith. Bioresource technology, 2011. 102(22): p. 10239-10247.

7. Mamisahebei, S., et al., Removal of arsenic from an aqueous solution by pretreated waste tea fungal biomass. Journal of Environmental Health Science \& Engineering, 2007. 4(2): p. 85-92.

8. Hristovski, K.D., H. Nguyen, and P.K. Westerhoff, Removal of arsenate and 17 $\alpha$-ethinyl estradiol (EE2) by iron (hydr) oxide modified activated carbon fibers. Journal of Environmental Science and Health Part A, 2009. 44(4): p. 354-361.

9. Roy, P., Modeling of arsenic removal from drinking water through fixed bed column operation by law cost adsorbeast. 2015.

10. Chowdhury, M. and R. Islam, Removal of Arsenic from Contaminated Water by Granular Activated Carbon Embedded with Nano scale Zero-valent Iron, 2015, Concordia University.

11. Madzvamuse, A., A STUDY OF THE REMOVAL OF Pb, Cd, AND Cr HEAVY METALS FROM AQUEOUS SOLUTIONS BY MORINGA OLIEFERA SEEDS IN COMPARISON TO CARBONIZED MORINGA OLIEFERA SEED. 2013.

12. Gottipati, R., Preparation and characterization of microporous activated carbon from biomass and its application in the removal of chromium (VI) from aqueous phase, 2012. 